\title{
$\checkmark$ Alterações do Ritmo c Circadiano no Atleta
}

Dr. Fernando Silva ${ }^{1}$, Dr. Manuel Vaz $z^{2,4,5}$, Dr. José Carlos Carneiro ${ }^{2,3,4}$

${ }^{1}$ Interno Formação Específica em Medicina Geral e Familiar - USF S. Tomé / ACES Grande Porto I - Santo Tirso/Trofa; ${ }^{2}$ Assistente Hospitalar em Pneumologia; ${ }^{3}$ Especialista em Medicina Desportiva; ${ }^{4}$ Centro Hospitalar do Médio Ave, E.P.E.; ${ }^{5}$ Centro de Medicina Desportiva de Guimarães.

\section{RESUMO / ABSTRACT}

O sono é muito importante para o bom funcionamento do organismo humano e é constituído por vários estádios. Neste texto abordar-se-ão os conceitos de ritmos biológicos, o ritmo circadiano e a temperatura corporal, que é considerada o principal marcador biológico do ritmo circadiano. Os seus distúrbios têm influência no rendimento do atleta, sendo abordado o jet leg. O diagnóstico destes distúrbios pode ser efetuado através de vários métodos subjetivos e objetivos. Finalmente, serão referidas estratégias de adaptação, abordando-se o exercício físico, a dieta e a medicação, entre outras.

Sleep is very important for the proper functioning of the human body and consists of several stages. This text will address the concepts of biological rhythms, the circadian rhythm and body temperature, which is considered the main biological marker of the circadian rhythm. Their disorders have an influence on the athlete's performance, with jet leg being approached. The diagnosis of these disorders can be made through various subjective and objective methods. Finally, adaptation strategies will be mentioned, addressing physical exercise, diet, and medication, among others.

\section{PALAVRAS-CHAVE / KEYWORDS}

Sono, ritmos biológicos, ritmo circadiano, jet leg

Sleep, biological rhythms, circadian rhythm, jet leg

\section{Introdução}

O sono é definido como um estado reversível que envolve uma separação percetual do ambiente associado a uma incapacidade relativa de resposta a estímulos. ${ }^{1}$ De acordo com a American Academy of Sleep Medicine (AASM), os adultos têm necessidade de dormir entre 7 e 9 horas(h), enquanto os adolescentes requerem sono adicional, idealmente entre as 8 e as 10h. A quantidade necessária de sono varia amplamente entre indivíduos e pode alterar de dia para dia dentro do mesmo indivíduo, sendo influenciado pela doença, falta de sono e stresses psicológicos ou fisiológicos. ${ }^{2}$

Estudos revelam que aproximadamente um terço da vida humana é passada a dormir. ${ }^{3}$ Num período de 24h, o corpo continuamente alterna entre os estádios de vigília e sono. ${ }^{4}$ Os estádios do sono podem ser discriminados através de um EEG por alterações na atividade elétrica do cérebro. Numa noite normal de sono, aproximadamente a cada 90 minutos o cérebro oscila entre dois estádios principais: Nonrapid Eye Movement (NREM) e Rapid Eye
Movement (REM), com cerca de 75\% do tempo passado em NREM. ${ }^{5}$

Dentro do NREM existem quatro estádios progressivamente mais profundos. ${ }^{6} \mathrm{O}$ estádio 1 é responsável pela transição entre estar acordado e a dormir e é caracterizado por resposta diminuída a estímulos externos. ${ }^{4}$ Aproximadamente 50\% de todo o tempo a dormir é passado no Estádio 2 e, mesmo que mais profundo que o estádio 1, ainda não é classificado como sono restaurador. ${ }^{4}$ Os estádios 3 e 4, que ocupam $15-20 \%$ de todo o tempo de sono, são comummente agrupados e referidos como delta, SWS ou sono profundo. $4,7,8$ Durante o REM o cérebro exibe um padrão de atividade neuronal similar ao observado aquando acordado, associando-se ao processo de consolidação e aprendizagem de habilidades motoras. A quantidade e qualidade de sono na noite posterior a um exercício de memória tem sido positivamente correlacionado com a extensão de recordar e reter no dia seguinte. ${ }^{9}$
O ser humano é parte integrante da natureza e a sua evolução foi propiciada pelo seu ajuste ao meio ambiente. ${ }^{10}$ Os ritmos biológicos referem-se a mudanças cíclicas repetidas num determinado tempo e relacionados com alterações dos processos fisiológicos. ${ }^{9}$

A cronobiologia é a ciência que estuda os mecanismos dos relógios biológicos, assim como as potenciais consequências da disrupção destes sistemas. ${ }^{11}$ Divide-se em cronofarmacologia e a cronoterapia, as quais estudam o timing ideal para a medicação a fim de melhorar a atividade metabólica com a máxima eficácia e reduzir os efeitos colaterais. A crononutrição estuda a relação entre a dieta e o ritmo circadiano. ${ }^{11}$ Mais recentemente, foi criado o termo de cronobiologia do desporto que estuda a perícia física e as suas respetivas funções bioquímicas, fisiológicas e psicológicas num determinado espaço temporal com particular foco nos mecanismos adaptativos e timings da performance, procurando a otimização ou eliminação de potenciais fatores de dessincronização. ${ }^{12}$

Os ciclos são denominados de circadianos, se referentes ao dia solar $(24+/-4 h)$, ultradianos (ciclos com menos de 24h) ou infradianos (ciclos com mais de 28h). ${ }^{13} \mathrm{O}$ ritmo circadiano é há muito reconhecido como regulador de múltiplos processos fisiológicos, nomeadamente o ciclo sono-vigília, a temperatura corporal, os padrões de atividade e a secreção de hormonas. ${ }^{11}$

O relógio biológico está localizado no hipotálamo, mais concretamente no núcleo supraquiasmático (SCN). O papel regulatório do SCN é alcançado recebendo informação diretamente de vias nervosas (ex: via retino-hipotalámica) e hormonais e indiretamente através da temperatura corporal, atividade física ou dieta. ${ }^{11}$ A nível molecular, a regulação do relógio circadiano assenta numa série de loops de feedback interligados (figura 1). ${ }^{14}$ Os genes chave envolvidos no sistema circadiano são chamados genes relógio. ${ }^{15}$ Os genes relógio de feedback positivo, BMAL1 e CLOCK, iniciam a expressão de genes de Per e Cry que, por 
sua vez, atuam na inibição da expressão de BMAL1/CLOCK bloqueando a sua própria expressão num loop negativo de feedback. Apenas quando a expressão de genes inibidores desce abaixo do nível limite, a expressão de "genes relógio" positivos é reativada. ${ }^{16}$ Este padrão de onda sinusoidal, resultante da coordenada subida e queda de "genes relógio", permite o controlo de processos jusante, resultando em efeitos fisiológicos específicos a horas específicas do ciclo sono-vigília. ${ }^{17}$

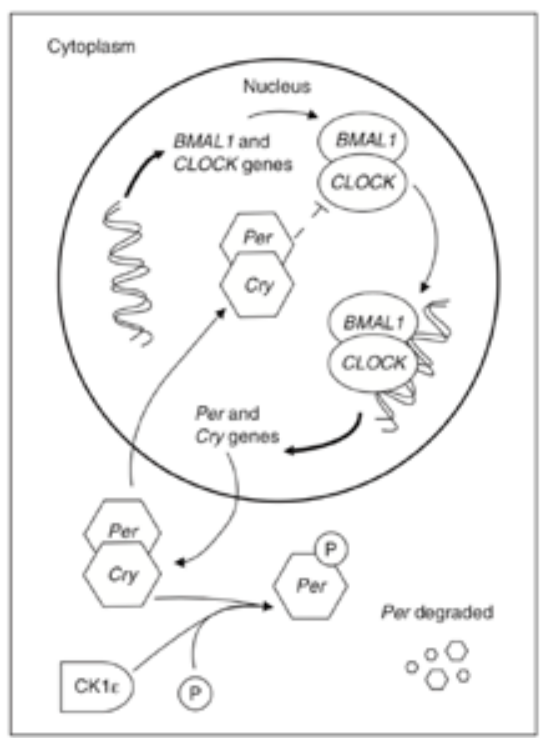

Figura 1 - Regulação molecular do relógio circadiano

A informação obtida do exterior, também denominada na literatura como zeitgeber, é processada centralmente e enviada para outros relógios periféricos presentes em tecidos e/ ou células. ${ }^{16} \mathrm{O}$ zeitgeber mais importante é a luz solar; outros zeitgebergs menos importantes incluem dieta, exercício, sono e tratamentos hormonais. ${ }^{17}$

A temperatura corporal é considerada o principal marcador biológico do ritmo do circadiano. Regulada por um grupo de células na porção anterior do hipotálamo, esta assume algumas características básicas: um mínimo pela manhã por volta das 4 h e começa a aumentar até atingir a sua acrofase por volta das 18h. ${ }^{9}$ Diversos autores sugerem que a capacidade de desempenho está intimamente relacionada com a hora em que a temperatura corporal atinge o seu pico. ${ }^{18} \mathrm{O}$ aumento da temperatura ao fim da tarde tem sido ligado ao aumento na coordenação, menor tempo de reação, aumento da força muscular e flexibilidade das articulações e eficiência cardiovascular. ${ }^{19}$

Outros marcadores também influenciados pelos ritmos biológicos incluem a melatonina e o cortisol plasmático. A melatonina é uma hormona secretada, em condições normais, entre as 21 e as $7 \mathrm{~h}$ pela glândula pineal. ${ }^{9}$ Durante o dia a sua atividade é inibida pelo SCN através de neurónios GABAérgicos, enquanto à noite neurónios glutaminérgicos estimulam a libertação. ${ }^{11}$ Além de induzir sono, acredita-se que a melatonina seja responsável pelo controlo de alguns dos ritmos circadianos. ${ }^{20}$

\section{Distúrbios do ritmo circadiano}

Tal como na população geral, as doenças do sono no atleta muitas vezes permanecem por diagnosticar, sendo que aquelas que mais podem afetar a performance do atleta são: síndrome da apneia obstrutiva do sono, insónia, síndrome das pernas inquietas e doenças do ritmo circadiano. ${ }^{21}$

O ritmo circadiano controla funções que são essenciais no atleta, como o alerta, a concentração, a força e a coordenação. O maior funcionamento é espectável que aconteça ao início da noite durante os picos do circadiano. As viagens frequentes, muitas das vezes através de múltiplos fusos horários, contribuem para a dessincronização do circadiano do atleta, resultando em jet lag. A gravidade aumenta de acordo com o número de fusos horários ultrapassados, mais de três zonas conduz inevitavelmente ao desenvolvimento desta condição. ${ }^{22}$ Os sintomas associados ocorrem enquanto o circadiano tenta adaptar-se. Evolutivamente, este é um processo de ajuste lento, de dias ou semanas e não para ser efetuado de forma abrupta, como depois de uma viagem de avião. ${ }^{16}$ Foi estabelecido que para a adaptação satisfatória é necessário um dia por cada hora ultrapassada. As queixas variam individualmente, sendo as principais: a dificuldade em dormir, fadiga durante o dia, perda de apetite e fraca performance. ${ }^{23}$ Foi observado que equipas de basebol atuam pior se tiverem que viajar através de fusos horários e jogar, mesmo que esse mesmo jogo aconteça na condição de visitado. ${ }^{24}$ Uma vantagem no circadiano também foi descoberta na liga profissional de futebol americano, em que as equipas da Costa Oeste parecem ter vantagem face às equipas da Costa Este, visto que os jogos podem acabar depois da meia noite (hora da Costa Este) que é mais tarde que a maioria da hora de deitar dos jogadores da Costa Este. ${ }^{25}$ São também descritas

Tabela 1 - Compilação de estudos relacionando sono e exercício físico no atleta

\begin{tabular}{|c|c|c|c|c|c|c|}
\hline \multirow{2}{*}{ Estudo } & \multirow{2}{*}{$\begin{array}{l}\text { Número de } \\
\text { sujeitos }\end{array}$} & \multirow{2}{*}{$\begin{array}{l}\text { Desporto e Pre- } \\
\text { paração Física }\end{array}$} & \multirow{2}{*}{ Protocolo } & \multicolumn{3}{|c|}{ Resultados } \\
\hline & & & & TTS & ES\% & LS \\
\hline Juliff et $\mathrm{al}^{37}$ & 42 & Netball & $\begin{array}{l}\text { Competição à noite vs } \\
\text { Competição de manhã }\end{array}$ & - & SR & $\mathrm{SR}$ \\
\hline Fullagar et $\mathrm{al}^{38}$ & 16 & Futebol & $\begin{array}{l}\text { Competição à noite vs } \\
\text { dia de treino }\end{array}$ & - & SR & - \\
\hline Lastella et $\mathrm{al}^{39}$ & 21 & Ciclismo & Competição vs basal & - & SS & SS \\
\hline Oda and Shirakawa ${ }^{40}$ & 12 & Adultos saudáveis & $\begin{array}{l}\text { Exercício de alta } \\
\text { intensidade vs Sem } \\
\text { exercício }\end{array}$ & - & - & - \\
\hline O’Donnell et al ${ }^{41}$ & 11 & Netball & $\begin{array}{l}\text { Competição à noite vs } \\
\text { Noite anterior vs Noite } \\
\text { após competição }\end{array}$ & - & SS & SS \\
\hline O’Donnell et al ${ }^{42}$ & 10 & Netball & $\begin{array}{l}\text { Jogos à noite vs Treino } \\
\text { à noite vs Basal }\end{array}$ & - & - & $\begin{array}{l}\text { NS } \\
1-\end{array}$ \\
\hline Richmond et $\mathrm{al}^{43}$ & 10 & $\begin{array}{l}\text { Liga de futebol } \\
\text { Australiano }\end{array}$ & Jogos à noite vs Basal & - & SS & SS \\
\hline Sargent e Roach ${ }^{44}$ & 22 & $\begin{array}{l}\text { Liga de futebol } \\
\text { Australiano }\end{array}$ & $\begin{array}{l}\text { Jogo à noite vs Jogo } \\
\text { de dia }\end{array}$ & - & SS & SS \\
\hline Shearer et $\mathrm{al}^{45}$ & 28 & Râguebi & $\begin{array}{l}\text { Jogo à noite como } \\
\text { visitado vs Noite } \\
\text { referência }\end{array}$ & - & SS & SS \\
\hline
\end{tabular}

Legenda: melhoria, - decréscimo, SR: sem relatório, SS: sem significado, ES\%: eficiência do sono, LS: latência do sono, TTS: tempo total de sono (Adaptação de O’Donnell et al, 2018) 
consequências a longo prazo de jet lag frequente: défices cognitivos, depressão, maior incidência de alterações de humor, aumento do risco de cancro, infertilidade, doença cardiovascular e atrofia cerebral. ${ }^{26}$

Outro fator a ter em conta, e que poderá afetar o desempenho ideal, é a variabilidade individual ou cronotipo ${ }^{27}$ : alguns atletas podem ser do tipo 'cotovia' (aqueles cujo circadiano é ligeiramente mais curto que 24h com tendência a avançar), do tipo 'mocho' (aqueles mais facilmente ajustáveis por atraso) ou do tipo 'intermédio'.

O atleta, cuja performance está intrinsecamente afetada por muitas alterações nos índices de sono, torna-se um caso de estudo interessante para todos os envolvidos na área desportiva, e não só. Múltiplos estudos têm sido realizados e na Tabela 1 encontram-se alguns exemplos. Os dados indicam que durações mais longas do sono durante a competição foram acompanhadas de melhores classificações no torneio, não havendo qualquer diferença entre sexos. ${ }^{28}$ Foi também demonstrado que alterações no ambiente de treino afetou o sono nos atletas, devendo ser tomadas as medidas adequadas com vista à mitigação destes potenciais influenciadores de desempenho.

\section{Diagnóstico}

Vários métodos subjetivos e objetivos têm sido usados para medir o sono na população geral. ${ }^{28}$ Embora todos estes métodos sejam utilizados como medidas para a investigação do sono, nem sempre são passíveis de reprodução no contexto do atleta. ${ }^{28}$

A polissonografia é considerada o gold standard da avaliação objetiva do sono em atletas. ${ }^{29}$ Este método é usado para diagnosticar e avaliar o tratamento das doenças do sono e fornece a mais rigorosa avaliação dos estádios do sono e da sua fisiologia..$^{30}$

A actigrafia (fgura 2) por ser um método mais prático e versátil, torna o estudo do sono no atleta um processo mais exequível. Avalia objetivamente o sono e é um método geralmente aceite na literatura. ${ }^{31} \mathrm{Um}$ artigo de revisão demonstrou concordância de 91-93\% entre a polissonografia e a actigrafia em adultos saudáveis e de idades compreendi-

Os questionários e diários de sono têm sido usados para avaliar mutualmente a qualidade e quantidade do sono ${ }^{33}$, oferecendo a perceção do participante do tempo na cama acordado e a qualidade do sono (tabela 2). De acordo com um estudo realizado em 63 atletas masculinos profissionais de râguebi, o sono foi sobrestimado por uma média de 19,8 minutos, tornando o uso de medidas subjetivas de medição do sono aceitável quando a medição objetiva não pode ser efetuada. ${ }^{33}$ Os questionários PSQI e ESS têm sido utilizados com frequência na investigação do sono. ${ }^{28}$ Todavia, foi sugerido que estes questionários poderiam não ser sensíveis o suficiente para determinar os desafios únicos e diferenças nos hábitos de sono de um atleta. ${ }^{34}$ Baseado nestas
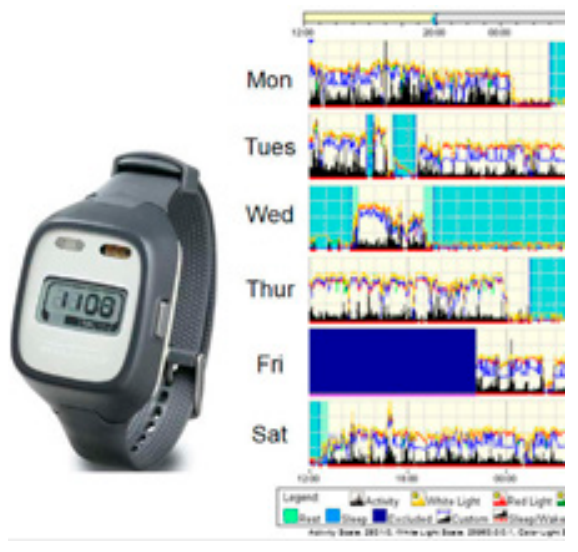

Figura 2 - Actigrafia (https://doctorferre. com/pruebas-del-sueno/actigrafia/) das entre os 20 e os 30 anos. ${ }^{32}$

dificuldades, surgiram o ASSQ e o ASBQ. O ASBQ é uma ferramenta mais prática que consiste num questionário com 18 itens e que pode ser usada por treinadores e equipa médica com a finalidade de identificação de comportamentos mal adaptativos da higiene do sono. ${ }^{34}$

\section{Estratégias de adaptação}

Atualmente, o ajuste do circadiano é algo com que muitos atletas se preocupam. Com o aprofundar dos estudos, novas estratégias foram surgindo para adaptações mais rápidas a dessincronizações do circadiano. A chave reside na educação dos atletas, reforçando a importância de bons hábitos e higiene no sono (tabela 3).

A decisão de tentar a adaptação ou não à hora local depende da duração da viagem. ${ }^{16}$ Para viagens pequenas (1 ou 2 dias) não é recomendado que se tente a adaptação ${ }^{35}$, já que o prazo é demasiado curto para se alcançar a adaptação completa pelos métodos convencionais e qualquer tentativa poderá ter um efeito adverso, piorando a situação. ${ }^{16}$ É sim recomendado que o atleta tente manter-se no seu fuso horário normal e que agende os eventos importantes a horas em que os níveis de alerta e performance sejam máximos. ${ }^{16}$ Todavia, em competições importantes, devido à pressão dos media, é raramente possível ajustar as horas do evento de forma a servir os interesses do atleta. ${ }^{16}$ Para viagens mais longas (>4-5 dias) podem ser designadas estratégias que visam apoiar a adaptação após a chegada ou, por outro lado, estas podem já ter sido implementadas antes da viagem. ${ }^{16}$ Teoricamente as estratégias baseiam-se no reforço dos zeitgebers, procurando uma mais rápida sincronização do circadiano ao fuso horário local. ${ }^{16}$

\section{Exercício e dieta}

\begin{tabular}{ll}
\hline Doenças do Sono & Questionários \\
\hline SAOS & STOP-Bang, Questionário de Berlim \\
\hline Síndrome das Pernas Inquietas & IRLSSG Criteria \\
\hline Insónia & Insomnia Severity Index \\
\hline Doença do Ritmo Circadiano & $\begin{array}{l}\text { Questionário de Horne-Ostberg, Questioná- } \\
\text { rio Morningness-Eveningness }\end{array}$ \\
\hline
\end{tabular}

(Adaptação de Malhotra R., 2017)
Apesar da pesquisa efetuada neste campo não ser conclusiva, acredita-se que a atividade física poderá afetar o circadiano. ${ }^{36} \mathrm{O}$ ajuste do horário dos exercícios de forma a coincidir com o pico do circadiano 
foi tentado e apresenta resultados promissores. O tipo de comida, assim como o horário das refeições, são também aspetos importantes no processo de adaptação. Sabe-se que a comida com alto teor de proteínas estimula a via da adrenalina, aumentando a preparação e alerta, sendo então recomendada a sua ingestão ao pequeno-almoço. ${ }^{11}$ Alimentos ricos em carboidratos devem ser ingeridos à noite, pois aumentam a secreção de insulina, facilitando a captação de triptofano, um aminoácido essencial precursor da serotonina que promove a sonolência. ${ }^{11}$ A hidratação adequada não pode ser esquecida porque elimina a fatiga que tradicionalmente acompanha o jet lag. ${ }^{37}$

\section{Cafeína}

O café é o único estimulante disponível para os atletas que não foi banido ou restringido pela Agência Mundial de Antidpagem. ${ }^{38} \mathrm{O}$ uso é permitido em doses baixas a moderadas ${ }^{39}$ e, apesar dos seus conhecidos efeitos deletérios, múltiplas investigações confirmaram a utilidade no campo da resistência ${ }^{40} \mathrm{e}$ velocidade. ${ }^{41}$

\section{Sesta}

A sesta é relatada como sendo uma medida comportamental usada para aliviar o débito de sono. ${ }^{42}$ Ocorre mais frequentemente durante a tarde, coincidindo com uma queda no ritmo circadiano. ${ }^{43}$ Tem sido destacado que existem duas durações ideais para uma sesta: sestas com duração inferior a 20 minutos reduzem a possibilidade de acordar durante o SWS ${ }^{42}$, como alternativa sestas de 90 minutos são também consideradas ótimas por permitir que um ciclo completo de sono (REM + NREM) ocorra. ${ }^{44}$

Tabela 3 - Medidas de higiene do sono

\begin{tabular}{|c|}
\hline Manter um horário de acordar e deitar constante \\
\hline Evitar luzes 1 a 2 horas antes do deitar (incluindo aparelhos eletrónicos) \\
\hline Procurar um lugar calmo e confortável para dormir \\
\hline Evitar álcool e café depois de jantar \\
\hline $\begin{array}{l}\text { Usar a cama apenas para dormir e sexo. Não praticar outras atividades, como comer ou ver } \\
\text { televisão }\end{array}$ \\
\hline $\begin{array}{l}\text { Evitar sestas longas (>30min) durante o dia, já que pode tornar mais complicado adormecer } \\
\text { à noite }\end{array}$ \\
\hline
\end{tabular}

\section{Luz solar}

A luz é um dos estímulos naturais mais fortes para a definição do circadiano, sendo capaz de controlar intrinsecamente a direção e velocidade de ajuste. ${ }^{16}$ Uma das estratégias mais simples consiste na promoção ou restrição de luz solar a horas específicas. ${ }^{16} \mathrm{~A}$ exposição deve ser cronometrada cuidadosamente para evitar efeitos adversos. ${ }^{16} \mathrm{~A}$ luz artificial, apesar de possuir apenas um terço da eficiência da luz solar ${ }^{45}$, também pode ser usada para a adaptação mais rápida. ${ }^{16}$ Estudos mostraram que pulsos de luz artificial num intervalo tão curto quanto 15 minutos poderão alterar o circadiano. ${ }^{46} \mathrm{O}$ circadiano é mais afetado pela luz azul devido à presença de melanopsina na retina ${ }^{16}$ e ao efeito supressor na secreção de melatonina. ${ }^{47}$ Para ambientes mais escuros, a luz verde parece ser mais efetiva que a azul. ${ }^{48}$

\section{Melatonina}

A melatonina permanece como um dos principais auxílios na prevenção e tratamento do jet lag. Foram demonstrados efeitos de sinergismo quando usada em combinação com a terapia de luz. ${ }^{49}$ As doses aconselhadas variam entre $05 \mathrm{mg}$ e $5 \mathrm{mg}$, sendo que formulações de atuação imediata parecem ser as mais eficazes. ${ }^{50}$ Para atrasos no curso do sono, é recomendado o seu uso numa fase mais tardia do sono ou durante a manhã, pois esta irá avançar o circadiano fazendo com que o corpo pense que a noite chegou mais cedo; para avanços do sono, é recomendada a sua administração à noite. ${ }^{51}$ Embora seja considerada segura e eficaz, deve ser administrada com cuidado em atletas, visto que em alguns países a sua compra e toma é proibida.
(Adaptação de Malhotra R., 2017)

\section{Sedativos e hipnóticos}

Os sedativos e os hipnóticos têm sido usados como segunda linha à melatonina. ${ }^{51}$ As medicações mais utilizadas têm sido os sedativos-hipnóticos não benzodiazepinas. As benzodiazepinas são desencorajadas pelo elevado potencial de abuso e consequente dependência. ${ }^{51}$ São caracterizados em longa duração (zopiclone e eszopiclone), curta-duração (zolpidem) e ultracurta duração (zeleplon) de acordo com a sua semi vida. ${ }^{51}$ Outros agentes, como a difenidramina, amitriptilina e trazodona, foram associados a sonolência excessiva no dia seguinte e, por esse motivo são raramente usados em atletas. ${ }^{52}$

\section{Conclusão}

Num meio tão competitivo e stressante como o da competição de elite, onde pequenos pormenores podem fazer a diferença entre o êxito e o fracasso, atletas e equipa médica procuram cada vez mais estratégias e/ou métodos que possam criar um elemento diferenciador. O sono surge como uma área da medicina ainda pouco estudada e por isso com muito potencial de intervenção, apesar de se já reconhecer o seu papel vital na otimização da performance desportiva, sabendo-se atualmente que o sono não adequado (em qualidade e quantidade) afeta desde a função neurocognitiva e imune até à esperança média de vida. Os défices de sono ou a sua fragmentação têm implicações em fundamentos básicos de sucesso num atleta: estado cognitivo, humor, tempo de reação e destreza musculoesquelética.

Uma vez que as doenças do sono ou somente sintomas são muitas vezes identificados e ignorados deveria ser reforçada a educação dos atletas e profissionais de saúde com vista a uma intervenção mais segura e dirigida à saúde no desporto.

\section{Correspondência}

Dr. José Carlos Carneiro -

dr.josecarneiro@gmail.com

Os autores declaram ausência de conflitos, assim com a originalidade do manuscrito e a sua não publicação prévia. 


\section{Bibliografia}

1. Carskadon, MA, Dement, WC. Normal human sleep: an overview. In: Principles and Practices of Sleep Medicine, 5th ed. Canada: Elsevier Saunders, 2011.

2. Watson NF, Badr MS, et al. Joint Consensus Statement of the American Academy of Sleep Medicine and Sleep Research Society on the recommended amount of sleep for healthy adult: methodology and discussion. Sleep. 2015; 38:1161-1183.

3. Fuller PM, Gooley JJ, Saper, CB. Neurobiology of the sleep-wake cycle: sleep architecture, circadian regulation, and regulatory feedback. J. Biol. Rhythms. 2006; 21:482-493.

4. Savis, J. Sleep and athletic performance: overview and implications for sport psychology. Sports Psychol. 1994; 8:111-125.

5. Bear MF, Connors BW, Paradiso, MA. Neuroscience: Exploring the Brain. Philadelphia: Lippincott Williams \& Wilkins, 2016.

6. Taylor SR, Rogers GG, Driver H.S. Effects of training volume on sleep, psycho-logical, and selected physiological profiles of elite female swimmers. Med. Sci. Sports Exerc. 1997; 29:688-690.

7. Davenne, D. Sleep of athletes-problems and possible solutions. Biol. Rhythm. Res. 2009; 40: 45-52.

8. Martini, F. Fundamentals of Anatomy and Physiology. 511h ed. Harlow: Pearson, 2017.

9. Minati, A.; Santana, M.G.; Mello, M.T. A influência dos ritmos circadianos no desempenho físico. R. Bras. Ci e Mov. 2006; 14(1):75-86.

10. Reilly T, Atkinson G, Waterhouse J. Chronobiology and physical performance. In: Garret WE., Kirkendall DT (ed). Exercise and sport science. 1st ed. Philadelphia: Williams \& Wilkins, 2000. Cap.24, p.351-372.

11. Vitosevic B. The Circadian clock and Human Athletic Performance. Natur Sciences. 2017; 7(1): 1-7.

12. Jancokova, L. Chronobiology from theory to sports practice. Krakow: Towarzystwo Slowakow w Posce, 2013.

13. Bird SP. Sleep, recovery, and athletic performance: a brief review and recommendations. Strength Cond. J. 2013; 35(5):43-47.

14. Hastings M, O'Neill JS, Maywood ES. Circadian clocks: regulators of endocrine and metabolic rhythms. J Endocrinol. 2007; 195(2):187-98.

15. Gekakis N, Staknis D, Nguyen HB, et al. Role of the CLOCK protein in the mammalian circadian mechanism. Science 1998; 280(5369):1564-1569.

16. Forbes-Robertson S., Dudley E., Vadgama P, Cook C, Drawer S and Kilduff L. Circadian Disruption and Remedial Interventions: effects and interventions for jet lag for athletic peak performance. Sports Med. 2012: 42(3):185-208.

17. Hofstra WA, de Weerd AW. How to assess circadian rhythm in humans: a review of literature. Epilepsy Behav.2008; 13(3):438-44.

18. Carter H, Jones AM, Maxwell NS, Doust JH. The effect of interdian and diurnal variation on oxygen uptake kinetics during treadmill running. J Sports Sci. 2002; 20:901-909.

19. Atkinson, G., \& Reilly, T. Circadian variation in sports performance. Sports Med. 1996;21(4): 292-312.

20. Bailey SL, Heitkemper MM. Circadian rhythmicity of cortisol and body temperature: morning - eveningness effects. Chronobiol Int 2001; 18(2):249-261.

21. Malhotra, R. Sleep, Recovery and Performance in Sports. Neurol Clinics. 2017; 35(3):547-557.

22. Jackson G. Come fly with me: jet lag and melatonin. Int J Clin Prac. 2010; 64(2):135-137.

23. Shneerson JM. Sleep medicine: a guide to sleep and its disorders. 2nd ed. Oxford: Blackwell, 2005.

24. Winter WC, Potenziano BJ, Zhang Z, et al. Chronotype as a predictor of performance in major league baseball batters. Sleep. 2011; 34: 167-168.

25. Eagles A, Mclellan C, Hing W, et al. Changes in sleep quantity and efficiency in professional rugby union players during home-based training and match play. J Sports Med Phys Fitness. 2016; 56(5):565-571.

26. Scott AJ. Shift work and health. Prim Care. 2000; 27(4):1057-79

27. O’Donnell S, Beaven C., Driller M. From pillow to podium: a review on understanding sleep for elite athletes. Nat Sci Sleep. 2018; 10:243-253.

28. Manfredini R, Manfredini F, Fersini C, et al. Circadian rhythms, athletic performance, and jet lag. Br J Sports Med. 1998; 32 (2):101-6.

29. Roky R, Herrera C, Ahmed Q. Sleep in athletes and the effects of Ramadan. J Sports Sci. 2012; 30(1):S75-S84.

30. Kushida C, Littner M, Morgenthaler T, et al. Practice parameters for the indications for polysomnography and related procedures: an update for 2005. Sleep. 2005; 28(4):499-520.

31. Driller M, McQuillan J, O’Donnell S. Inter-device reliability of an automatic-scoring actigraph for measuring sleep in healthy adults. Sleep Sci. 2016; 9(3):198-201.

32. Ancoli-Israel S, Cole R, Alessi C, Chambers M, Moorcroft W, Pollak C. The role of actigraphy in the study of sleep and circadian rhythms. Sleep. 2003; 26(3):342-392.

33. Caia J, Thornton H, Kelly V, et al. Does self-perceived sleep reflect sleep estimated via activity monitors in professional rugby league athletes? J Sports Sci. 2018; 36(13):1492-1496.

34. Driller M, Mah C, Halson S. Development of the athletes sleep behavior questionnaire: a tool for identifying maladaptive sleep practices in elite athletes. Sleep Sci. 2018; 11(1):37-44

35. Arendt J. Managing jet lag: some of the problems and possible new solutions. Sleep Med Rev. 2009; 13(4):249-256.

36. Mistlberger RE. Scheduled daily exercise or feeding alters the phase of photic entrainment in Syrian hamsters. Physiol Behav 1991; 50 (6):1257-1260.

37. Auger RR, Morgenthaler TI. Jet lag and other sleep dis - orders relevant to the traveler. Travel Med Infect Dis. 2009; 7(2):60-68.

38. Baird M., Asif F. Medications for Sleep Schedule Adjustments in Athletes. Sports Health. 10(1):35-39.

39. Spriet LL, Graham TE. ACSM current comment. 2015 (http://www.acsm.org; accessed November 27, 2019)

40. Cook C, Beaven CM, Kilduff LP, Drawer S. Acute caffeine ingestion's increase of voluntarily chosen resistance-training load after limited sleep. Int J Sport Nutr Exerc Metab. 2012; 22:157-164.

41. Lara B, Ruiz-Vicente D, Areces F, et al. Acute consumption of a caffeinated energy drink enhances aspects of performance in sprint swimmers. Br J Nutr. 2015; 114:908-914.

42. Petit E, Mougin F, Bourdin H. A 20-min nap in athletes changes subsequent sleep architecture but does not alter physical performances after normal sleep or 5-h phase-advance conditions. Eur J Appl Physiol. 2014; 114(2):305-315.

43. Waterhouse J, Atkinson G, Edwards B, Reilly $\mathrm{T}$. The role of a short post-lunch nap in improving cognitive, motor, and sprint performance in participants with partial sleep deprivation. J Sports Sci. 2007; 25(14):1557-1566.

44. Davies D, Graham K, Chow C. The effect of prior endurance training on nap sleep patterns. Int J Sports Physiol Perform. 2010; 5(1):87-97.

45. Boivin DB, Duffy JF, Kronauer RE, et al. Dose-response relationships for resetting of human circadian clock by light. Nature. 1996; 379(6565): 540-2.

46. Gronfier C, Wright Jr KP, Kronauer RE, et al. Efficacy of a single sequence of intermittent bright light pulses for delaying circadian phase in humans. Am J Physiol Endocrinol Metab 2004; 287(1):E174-81.

47. Lockley SW, Brainard GC, Czeisler CA. High sensitivity of the human circadian melatonin rhythm to resetting by short wavelength light. J Clin Endocrinol Metab. 2003; 88(9):4502-5.

48. Arendt J. Managing jet lag: some of the problems and possible new solutions. Sleep Med Rev. 2009; 13(4):249-56.

49. Paul MA, Gray GW, Lieberman HR, et al. Phase advance with separate and combined melatonin and light treatment. Psychopharmacology. 2010; 214:515-523.

50. Herxheimer A, Petrie KJ. Melatonin for the prevention and treatment of jet lag. Cochrane Database Syst Rev. 2002; (2):CD001520.

51. Van Ryswyk E, Weeks R, Bandick L, et al. A novel sleep optimization program to improve athletes' well-being and performance. Eur J Sport Sci. 2017; 17(2):144-151.

52. Kay GG, Schwartz HI, Wingertzahn MA, Jayawardena S, Rosenberg RP. Next-day residual effects of gabapentin, diphenhydramine, and triazolam on simulated driving performance in healthy volunteers: a phase 3, randomized, double blind, placebo-controlled, crossover trial. Hum Psychopharmacol. 2016; 31:217-226. 